\title{
Performance Evaluation of FM-COOK Chaotic Communication System
}

\author{
Hikmat N. Abdullah ${ }^{1}$, Alejandro A. Valenzuela ${ }^{2}$ \\ ${ }^{1}$ Department of Electrical Engineering, Al-Mustansiryah University, Baghdad, Iraq; ${ }^{2}$ Department of Electrical Engineering, Mecha- \\ nical Engineering and Technical Journalism, University of Applied Sciences Bonn-Rhein-Sieg, Bonn, Germany. \\ Email: \{hikmat.abdullah, alejandro.valenzuela\}@h-brs.de
}

Received May $3^{\text {rd }}, 2011$; revised May 30 ${ }^{\text {th }}, 2011$; accepted June $7^{\text {th }}, 2011$.

\begin{abstract}
In this paper, the performance evaluation of Frequency Modulated Chaotic On-Off Keying (FM-COOK) in AWGN, Rayleigh and Rician fading channels is given. The simulation results show that an improvement in BER can be gained by incorporating the FM modulation with COOK for SNR values less than $10 \mathrm{~dB}$ in AWGN case and less than $6 \mathrm{~dB}$ for Rayleigh and Rician fading channels.
\end{abstract}

Keywords: Chaotic Transceiver, On-Off Keying, FM Modulation

\section{Introduction}

Chaotic signals are a relatively new field of interest in communication systems. Motivations of this method derived from the advantages offered by chaotic signals, such as robustness in multipath environments and resistance to jamming. Chaotic signals are non-periodic, broadband, and difficult to predict and reconstruct. These are properties which match with requirements for signals used in communication systems [1].

Various types of modulation can be used in direct chaotic communication systems like chaotic on-off keying (COOK), differential chaotic shift keying (DCSK) and additive chaos modulation (ACM), etc. Among all chaotic modulation types, DCSK with orthonormal basis functions offers the best robustness against multipath and channel imperfections [2]. Due to the length and non-periodicity of chaotic sample functions, the energy per bit is not constant and varies from one sample function to another. As a result, this varied energy per bit limits the noise performance of modulation system.

In 1998, Kolumbán et al. [3] show that FM-DCSK scheme generates an inherently wideband signal with constant energy per bit, which enhances the noise performance of DCSK. In 2001, Z. Galias and G. Maggio [4] proposed QCSK (Quadrature Chaos Shift Keying) scheme which can transmit 2 bits in a sample function to improve the speed of chaos shift keying. Then in 2006, Y. Zhang [5] devised FM-QCSK which enhanced the noise performance of QCSK by using frequency modulation, because it can generate constant energy per bit and its frequency spectrum. In 2009, J. Pan and H. Zhang [6] proposed an advanced version of the previous scheme named FM-QACSK (Frequency Modulated Quadrature Amplitude chaos shift keying) where 256QAM modulation is used to increase data rate also combined with the use of FM modulation.

Although the performance of COOK chaotic modulation lags behind the DCSK, the COOK have an advantage of that it consumes less power since it sends chaotic signal only in case of sending the data bit " 1 " while no power is sent for case of data bit " 0 ". However, we don't find in the literature the performance evaluation of the use of FM modulation with COOK to get constant energy per bit. So, this paper is an attempt to evaluate the performance of FM-COOK modulation scheme.

\section{FM-COOK Modulation}

Figure 1 shows the block diagram of FM-COOK system. Since the chaotic signal is nonperiodic and never repeated, the energy in each bit (for symbol "1") would vary from one bit to another. The variance of estimation can be reduced by increasing the statistical bandwidth of the transmitted chaotic signal or by increasing the bit duration [7]. Alternatively, one may solve the problem directly by modifying the generation of the basis functions such that the transmitted energy for each symbol is kept constant by applying the chaotic signal to an FM 
modulator. Recall that the instantaneous power of an FM signal does not depend on the modulation, provided that the latter is slowly varying compared to the carrier. Hence the transmitted FM-COOK is given by:

$$
s(t)=\left\{\begin{array}{lr}
A_{c} \cos \left[\omega_{c} t+K_{f} \int_{-\infty}^{\tau} c(\propto) \mathrm{d} \propto\right] & \text { when } \mathrm{d}(t)=1 \\
0 & \text { when } \mathrm{d}(t)=0
\end{array}\right.
$$

where $\mathrm{d}(t)$ is the transmitted data, $A_{c}$ is the carrier amplitude, $\omega_{c}$ is the carrier frequency, $K_{f}$ is the modulation constant in $\mathrm{Hz} / \mathrm{V}$ and $\mathrm{c}(t)$ is the chaotic signal. The average energy per bit is $A_{c}^{2}$.

The upper limit on the noise performance of a modulation scheme is determined by the separation of the message points in the signal space; the greater the separation, the better the noise performance. The distance between the message points in FM-COOK is $A_{c}^{2}$. In principle, the best noise performance in an AWGN channel can be achieved by using a coherent receiver [7]. In practice, the propagation conditions may be so poor that it is difficult, if not impossible, to regenerate the basis functions at the receiver. Under these conditions, a noncoherent receiver may offer better performance. The non-coherent detector shown in Figure $\mathbf{1}$ is, in fact, a bit-energy estimator whose output for the $l$ th symbol is given by:

$$
\begin{aligned}
y\left(1 T_{b}\right) & =\int_{(l-1) T_{b}}^{1 T_{b}} r^{2}(t) \mathrm{d} t=\int_{(l-1) T_{b}}^{1 T_{b}} s^{2}(t) \mathrm{d} t \\
& +2 \int_{(l-1) T_{b}}^{1 T_{b}} s(t) n(t) \mathrm{d} t+\int_{(l-1) T_{b}}^{1 T_{b}} n^{2}(t) \mathrm{d} t
\end{aligned}
$$

where $n(t)$ is the noise signal and $r(t)$ is the received signal plus noise. In the absence of the noise signal $n(t)$, the bit-energy estimator gives:

$$
s(t)=\left\{\begin{aligned}
& \int_{(l-1) T_{b}}^{1 T_{b}} A_{c}^{2} \cos ^{2}\left[\omega_{c} t+K_{f} \int_{-\infty}^{t} c(\propto) \mathrm{d} \propto\right] \\
& \text { when } \mathrm{d}(t)=1 \\
& 0 \text { when } \mathrm{d}(t)=0
\end{aligned}\right.
$$

The output is then passed to the threshold detector with the threshold set to mid-value of

$\left[E \int_{(l-1) T_{b}}^{1 T_{b}} A_{c}^{2} \cos ^{2}\left[\omega_{c} t+K_{f} \int_{-\infty}^{t} c(\propto) \mathrm{d} \propto\right]\right]$ and zero, where

$E[$.$] denotes the expectation operator. The decoded sym-$ bol is " 1 " if the estimated bit energy is larger than the threshold, otherwise a " 0 " is recovered. In the presence of noise (and fading), the threshold level needs to be shifted in order to optimize the performance. Note that

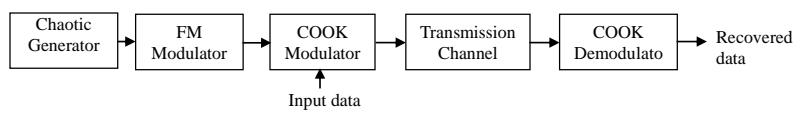

Figure 1. The block diagram of FM-COOK system. no FM demodulator is needed in the receiver because it is only use to get constant energy per bit.

\section{Simulation Results}

A simulation model has been implemented for FMCOOK chaotic modulator. Hennon mapping given by: $x_{n+1}=1+b x_{n}+a x_{n}^{2}$ where $a$ and $b$ are constants and $a=$ -1.4 and $b=0.3$ is used to generates the chaotic signal. According to the article [8], we define the chip rate equals $0.05 \mu \mathrm{s}$ and $T_{b}=4 \mu \mathrm{sec}$. For FM modulator, $A_{c}=$ $1 \mathrm{~V}, f_{c}=36 \mathrm{MHz}$ and then up converted to $433 \mathrm{MHz}$ and $K_{f}=7.8 \mathrm{MHz} / \mathrm{v}$.

Figure 2 shows the noise performance of FM-COOK as compared with COOK in AWGN channel. It is clear from this figure that the performance of FM-COOK is better than traditional COOK up to SNR $=10 \mathrm{~dB}$ and after than the performance becomes similar. However, this improvement is unfortunately lies in the region where the error probability is less than $10^{-4}$. This is because the threshold detector can easily recognize the high energy level for bit " 1 ” as compared with bit " 0 ” for high values of SNR, so the use of FM modulation would not add extra improvement. Figures 3 and $\mathbf{4}$ show the performance in Rayleigh and Racian fading channels respectively. In this case we used in our simulations two paths, the second path delay was 75 ns with attenuation of $-3 \mathrm{~dB}$ which represents the specification of multipath environment inside office buildings. Here the performance of FM-COOK is better than COOK up to SNR $=6$ $\mathrm{dB}$ for both cases. The improvement region along the performance curves here is much worse than that in AWGN channel (BER less than $10^{-2}$ ).

However, these improvements can be increased relatively as the ratio of bit duration to chip duration decrease

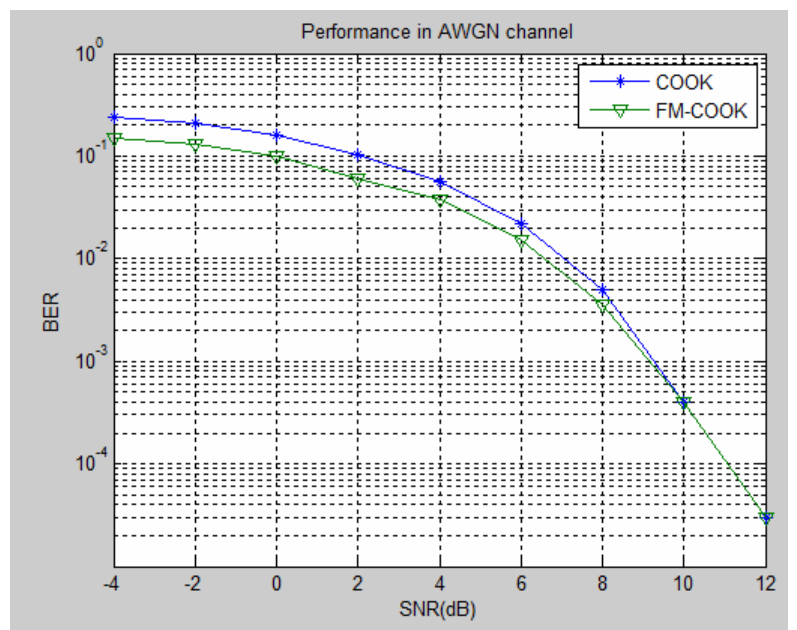

Figure 2. Performance of FM-COOK as compared with COOK in AWGN channel. 


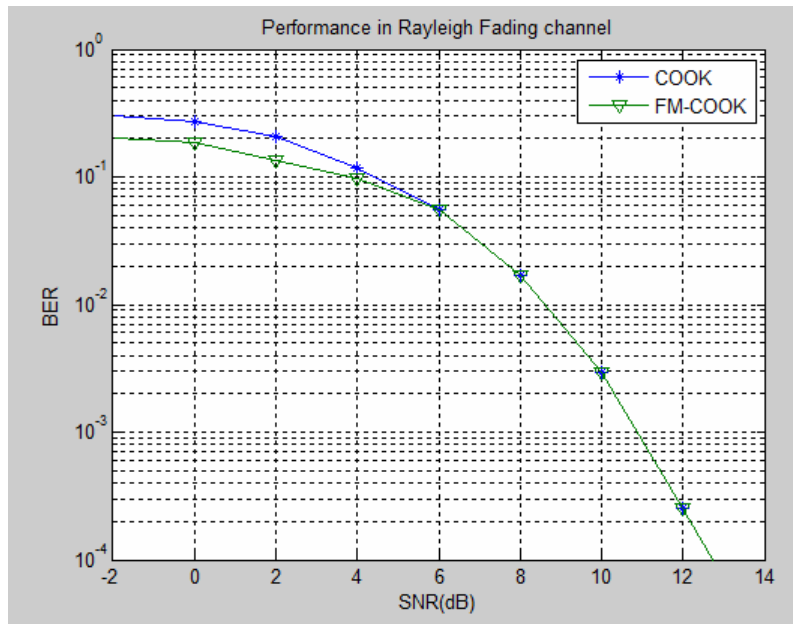

Figure 3. Performance of FM-COOK as compared with COOK in Rayleigh Fading channel.

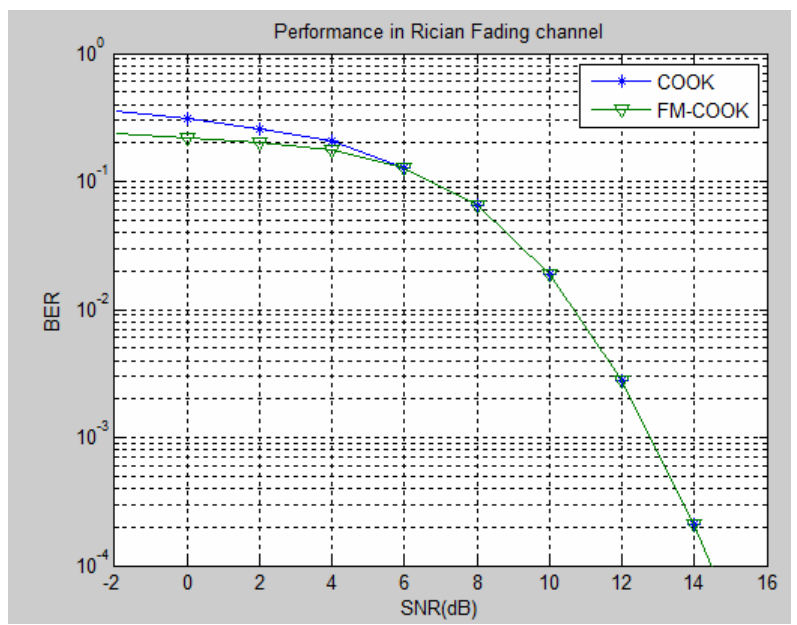

Figure 4. Performance of FM-COOK as compared with COOK in Rician Fading channel.

because we would get more variance in the energy level from one bit to another, so the use of FM would function well. But from other hand this would make the chaotic signal loss its characteristics as a spreading signal since the process gain of spreading operation would be greatly reduced which is not a practical case.

\section{Conclusions}

Although the FM-DCSK system offer the best noise performance in noisy and fading channels, the FM-COOK system offers the advantage of less power requirements which is important for some applications. The use FM modulation with COOK would provide constant energy per bit which leads to improve the noise performance in this system. Unfortunately, this improvement is achieved at low values of BER, so we think that the disadvantage of increasing the transmitter complexity by adding the FM modulator to COOK transmitter is considered more than the advantage gained in BER from practical point of view.

\section{Acknowledgements}

The authors would like to thank IIE organization for financing support to this work.

\section{REFERENCES}

[1] G. Kaddoum and F. Gagonen, "Error Correction Codes for Secure Chaos-Based Communication System," 25th Biennial Symposium on Communication, Kingston, 12-14 May 2010, pp. 193-196. doi:10.1109/BSC.2010.5472918

[2] G. Kolumbán, B. Vizvári, W. Schwarz and A. Abel, "Differential Chaos Shift Keying: A Robust Coding for Chaotic Communication," Proceedings of NDES'96, Seville, 27-28 June 1996, pp. 87-92.

[3] G. Kolumbán, G. Kis, Z. Jákó and M. P. Kennedy, “FMDCSK: A Robust Modulation Scheme for Chaotic Communications," IEICE Transactions on the Fundamentals, Vol. E81-A, October 1998, pp. 1798-1802.

[4] Z. Galias and G. M. Maggio, "Quadrature Chaos-Shift Keying: Theory and Performance Analysis,” IEEE Transactions on Circuits and Systems, Vol. 48, No. 12, 2001, pp. 1510-1518.

[5] Y. Zhang, X. Shen and Y. Ding, "Design and Performance Analysis of an FM-QCSK Chaotic Communication System,” 2nd International Conference on Wireless Communications, Networking and Mobile Computing, Wuhan, 22-24 September 2006, pp. 1-4.

[6] J. Pan and H. Zhang, "Design of FM-QACSK Chaotic Communication System,” International Conference on Wireless Communications \& Signal Processing, Nanjing, 13-15 November 2009, pp. 1184-1187.

[7] G. Kolumbán, et al., "Chaotic Communications with Correlator Receivers: Theory and Performance Limits," Proceedings of IEEE, Vol. 90, No. 5, 2002, pp. 711-732.

[8] M. A. B. Faran, A. Kachouri and M. Samet, "Design of Secure Digital Communication Systems Using DCSK Chaotic Modulation,” DTIS 2006 International Conference on Design and Test of Integrated Systems in Nanoscale Technology, Tunis, 5-7 September 2006, pp. 200204. 